\title{
Hyperbolic homeomorphisms and bishadowing
}

\author{
by P. E. Kloeden (Geelong, Victoria) and J. Ombach (Kraków)
}

\begin{abstract}
Hyperbolic homeomorphisms on compact manifolds are shown to have both inverse shadowing and bishadowing properties with respect to a class of $\delta$-methods which are represented by continuous mappings from the manifold into the space of biinfinite sequences in the manifold with the product topology. Topologically stable homeomorphisms and expanding mappings are also considered.
\end{abstract}

1. Introduction. The shadowing or pseudo-orbit tracing property of a dynamical system is often used to justify the validity of computer simulations of the system, asserting that there is a true orbit of the system close to the computed pseudo-orbit. The property was first established for systems generated by hyperbolic diffeomorphisms and later for those generated by hyperbolic homeomorphisms $[1,7,9,10,11]$.

In numerical calculations an inverse form of the shadowing concept is also of some interest: can every orbit of the system be shadowed by a numerical trajectory calculated by the specific computational routines and procedures under consideration? A composite concept of bishadowing, combining both direct and inverse shadowing, was proposed by Diamond et al. $[6,4,5]$ and shown to hold for systems generated by semi-hyperbolic Lipschitz mappings with the pseudo-orbits being true orbits of nearby continuous mappings. An appropriate choice of the class of admissible pseudo-orbits is crucial here, for Corless and Pilyugin [2] have shown that diffeomorphisms satisfying a strong transversality condition are not inverse shadowing if this class is too large.

Shadowing and hyperbolicity, or some modification of hyperbolicity, in a dynamical system are closely entwined. Indeed, it is now known [9] that hyperbolic homeomorphisms on a compact manifold as defined by Mañé [7] are characterized equivalently by expansivity and the shadowing property. In this paper it will be shown that such mappings also have inverse shad-

1991 Mathematics Subject Classification: Primary 58F15; Secondary 54H20.

Key words and phrases: pseudo-orbit, hyperbolic, shadowing.

Partially supported by the Australian Research Council Grant A 89132609. 
owing and bishadowing properties with respect to a class of pseudo-orbits corresponding to "methods" which are represented by continuous mappings from the manifold into the space of bi-infinite sequences in the manifold with the product topology. Definitions are presented in Section 2 and the result formulated as a theorem and proved in Section 3. Finally, in Section 4 it is briefly shown that the result also extends to topologically stable mappings and to expanding mappings.

2. Shadowing, inverse shadowing and bishadowing. Let $(X, d)$ be a compact metric space with metric $d$ and let $f: X \rightarrow X$ be a homeomorphism of $X$ onto itself.

A sequence $\left\{x_{n}\right\}_{n \in \mathbb{Z}}$ is called an orbit of $f$ if $x_{n+1}=f x_{n}$ for all $n \in \mathbb{Z}$, and a $\delta$-pseudo-orbit of $f$ if

$$
d\left(f x_{n}, x_{n+1}\right) \leq \delta \quad \text { for all } n \in \mathbb{Z} .
$$

We say that the homeomorphism $f$ has the shadowing property if for each $\varepsilon>0$ there exists $\delta>0$ such that every $\delta$-pseudo-orbit $\left\{y_{n}\right\}_{n \in \mathbb{Z}}$ is $\varepsilon$ shadowed by an orbit $\left\{f^{n} x\right\}_{n \in \mathbb{Z}}$ of $f$ for some $x \in X$, i.e.

$$
d\left(f^{n} x, y_{n}\right) \leq \varepsilon \quad \text { for all } n \in \mathbb{Z} .
$$

Recall that a homeomorphism $f$ is expansive if there exists a constant $\zeta>0$ such that $d\left(f^{n} x, f^{n} y\right) \leq \zeta$ for all $n \in \mathbb{Z}$ implies that $x=y$. Hyperbolic homeomorphisms [7] are expansive and also have the shadowing property. In fact, they are characterized by such properties, that is, a homeomorphism is hyperbolic if and only if it is expansive and has the shadowing property [9].

The concepts of inverse shadowing and bishadowing are relatively recent $[2,6,4,5,12]$. Let $X^{\mathbb{Z}}$ denote the compact space (with the product topology) of all two-sided sequences $\mathbf{x}=\left\{x_{n}\right\}_{n \in \mathbb{Z}}$ with components $x_{n} \in X$ and let $\boldsymbol{\Phi}_{f}(\delta) \subset X^{\mathbb{Z}}$ be the set of all $\delta$-pseudo-orbits of a homeomorphism $f$ for a given $\delta>0$. We call a mapping $\varphi: X \rightarrow \boldsymbol{\Phi}_{f}(\delta)$ a method of accuracy $\delta$ of $f$, or just a $\delta$-method for short, and note that the image of the space $X$ under a $\delta$-method is a complete family of $\delta$-pseudo-trajectories in the terminology of Corless and Pilyugin [2, 12].

EXAMPLE 1. The orbits of a one-to-one mapping $g: X \rightarrow X$ with $D_{\infty}(g, f)<\delta$, where $D_{\infty}(g, f)=\sup _{x \in X} d(g x, f x)$, is a $\delta$-method with $\varphi$ defined by $\varphi(x)=\operatorname{orbit}_{g}(x)$ for each $x \in X$.

A $\delta$-method need not, however, be generated by a single mapping.

Example 2. Consider two functions $g_{i}: X \rightarrow X$ with $D_{\infty}\left(g_{i}, f\right) \leq \delta$, $i=1,2$, and for each point $x \in X$ define a two-sided sequence $\varphi(x) \in \mathbf{\Phi}_{f}(\delta)$ by

$$
\varphi(x)=\left\{\ldots, f^{-2} x, f^{-1} x, x, g_{\alpha_{1}} x, g_{\alpha_{2}} g_{\alpha_{1}} x, g_{\alpha_{3}} g_{\alpha_{2}} g_{\alpha_{1}} x, \ldots\right\}
$$


with $\alpha_{1}, \alpha_{2}, \alpha_{3}, \ldots \in\{1,2\}$, where $x$ is the 0 th component. Such a $\delta$-method $\varphi$ arises when a two-step process is used to simulate the positive orbits of the dynamical system generated by $f$.

Let $\mathcal{T}$ be a family of methods containing the 0 -methods, i.e. methods generated by the homeomorphism $f$ itself.

Definition 1. A homeomorphism $f$ is said to be

(i) inverse shadowing with respect to the class $\mathcal{T}$ if for any $\varepsilon>0$ there is a $\delta>0$ such that for any $\delta$-method $\varphi \in \mathcal{T}$ and any point $y \in X$ there exists a point $x \in X$ for which

$$
d\left(f^{n} y, \varphi(x)_{n}\right) \leq \varepsilon \quad \text { for all } n \in \mathbb{Z}
$$

(ii) bishadowing with respect to the class $\mathcal{T}$ if for any $\varepsilon>0$ there is a $\delta \geq 0$ such that for any $\delta$-method $\varphi \in \mathcal{T}$ and any $\delta$-pseudo-orbit $\left\{y_{n}\right\}$ there exists a point $x \in X$ for which

$$
d\left(y_{n}, \varphi(x)_{n}\right) \leq \varepsilon \quad \text { for all } n \in \mathbb{Z} .
$$

Note that the definitions of shadowing and inverse shadowing are included in that of bishadowing by considering 0-methods and 0-pseudo-orbits, respectively, while an application of the triangle inequality shows that shadowing and inverse shadowing combined imply bishadowing.

In numerical applications it is important to know just how $\delta$ depends on $\varepsilon$ in the above definitions. In most proofs of the Shadowing Lemma this dependence often appears, implicitly at least, in the form $\delta \leq K \varepsilon$ for some constant $K$ provided $\varepsilon>0$ is sufficiently small. Such a relationship was used explicitly in $[6,4,5]$ by Diamond et al. to define inverse shadowing and bishadowing for semi-hyperbolic mappings, which they called $\alpha$-robustness and $(\alpha, \beta)$-bishadowing, respectively. We shall restate their definitions here in a slightly more general setting. Call

$$
D_{f}(\varphi)=\sup _{\substack{x \in X \\ k \in \mathbb{Z}}} d\left(f \varphi(x)_{k}, \varphi(x)_{k+1}\right)
$$

the 1-step deviation of a $\delta$-method $\varphi$ with respect to a homeomorphism $f$, so $D_{f}(\varphi) \leq \delta$, and note that $D_{f}(\varphi) \leq D_{\infty}(g, f)$ if the method $\varphi$ is generated by a single mapping $g$ as in Example 1 , while $D_{f}(\varphi) \leq \max \left(D_{\infty}\left(g_{1}, f\right), D_{\infty}\left(g_{2}, f\right)\right)$ if $\varphi$ is generated by two mappings $g_{1}$ and $g_{2}$ as in Example 2 .

Definition 2. Let $\alpha, \beta>0$ be fixed and let $\mathcal{T}$ be a family of methods. A homeomorphism $f$ is said to be

(i) $(\alpha, \beta)$-shadowing if for every $\delta$-pseudo-orbit $\left\{y_{n}\right\}$ with $\delta \leq \beta$ there exists a point $x \in X$ such that

$$
d\left(f^{n} x, y_{n}\right) \leq \alpha \delta \quad \text { for all } n \in \mathbb{Z}
$$


(ii) $(\alpha, \beta)$-inverse shadowing, or $(\alpha, \beta)$-robust, with respect to the class $\mathcal{T}$ if for every $\beta$-method $\varphi \in \mathcal{T}$ and every point $y \in X$ there exists a point $x \in X$ such that

$$
d\left(f^{n} y, \varphi(x)_{n}\right) \leq \alpha D_{f}(\varphi) \quad \text { for all } n \in \mathbb{Z} ;
$$

(iii) $(\alpha, \beta)$-bishadowing with respect to the class $\mathcal{T}$ if for every $\delta$-pseudoorbit $\left\{y_{n}\right\}$ and every $\beta$-method $\varphi \in \mathcal{T}$ with

$$
\delta+D_{f}(\varphi) \leq \beta
$$

there exists a point $x \in X$ such that

$$
d\left(y_{n}, \varphi(x)_{n}\right) \leq \alpha\left(\delta+D_{f}(\varphi)\right) \quad \text { for all } n \in \mathbb{Z} .
$$

Clearly, if a homeomorphism is $\left(\alpha_{1}, \beta_{1}\right)$-shadowing and $\left(\alpha_{2}, \beta_{2}\right)$-inverse shadowing with respect to some class of methods $\mathcal{T}$, then it is $(\alpha, \beta)$ bishadowing with respect to the same class $\mathcal{T}$ for $\alpha=\max \left(\alpha_{1}, \alpha_{2}\right)$ and $\beta=$ $\min \left(\beta_{1}, \beta_{2}\right)$. Moreover, $(\alpha, \beta)$-bishadowing implies both $(\alpha, \beta)$-shadowing and $(\alpha, \beta)$-inverse shadowing.

3. Main result. Since it has been shown by Corless and Pilyugin [2] that diffeomorphisms satisfying a strong transversality condition are not inverse shadowing with respect to the class of all possible methods, we restrict attention here to the class $\mathcal{T}_{\mathrm{c}}$ of all methods $\varphi$ that are continuous as functions from $X$ to $X^{\mathbb{Z}}$. Note that the methods in Examples 1 and 2 are in the class $\mathcal{T}_{\mathrm{c}}$ when $g$ is a homeomorphism and when $g_{1}$ and $g_{2}$ are continuous.

Our main result is

THEOREM 1. Let $X$ be a compact manifold and let $f: X \rightarrow X$ be a hyperbolic homeomorphism. Then $f$ is bishadowing with respect to the class of methods $\mathcal{T}_{\mathrm{c}}$. If in addition $f$ is $(\alpha, \beta)$-shadowing, then it is also $\left(\alpha, \beta_{1}\right)$ bishadowing for some sufficiently small $\beta_{1}>0$.

P r o of. To prove the first statement it is enough to show that $f$ is inverse shadowing. Let $\zeta>0$ be the expansiveness threshold constant for $f$, fix $\varepsilon$ $\leq \zeta / 2$ and choose $\delta$ corresponding to this $\varepsilon$ in the shadowing property of $f$.

Given any $\delta$-method $\varphi \in \mathcal{T}_{\mathrm{c}}$ we construct a map $h=h_{\varphi}: X \rightarrow X$ as follows. For each point $x \in X$ the sequence $\varphi(x) \in X^{\mathbb{Z}}$ is a $\delta$-pseudo-orbit. Let $h(x)$ be a point in $X$ which $\varepsilon$-shadows this $\delta$-pseudo-orbit, i.e. we have

$$
d\left(f^{n} h(x), \varphi(x)_{n}\right) \leq \varepsilon \quad \text { for all } n \in \mathbb{Z} .
$$

In fact, $h(x)$ is uniquely determined. To see this let $y \in X$ be a point for which the orbit also $\varepsilon$-shadows the $\delta$-pseudo-orbit $\varphi(x)$, i.e.

$$
d\left(f^{n} y, \varphi(x)_{n}\right) \leq \varepsilon \quad \text { for all } n \in \mathbb{Z} .
$$


By the triangle inequality and the choice of $\varepsilon$ we have

$$
d\left(f^{n} y, f^{n} h(x)\right) \leq 2 \varepsilon \leq \zeta \quad \text { for all } n \in \mathbb{Z} .
$$

Hence from the expansiveness of $f$ it follows that $y=h(x)$.

To show that the mapping $h$ is continuous if $\varepsilon$ is sufficiently small we shall use the following property of an expansive homeomorphism $[12,14]$. For $\eta>0$ and a natural number $N$ write

$$
B_{\eta}=\{(x, y) \in X \times X: d(x, y) \leq \eta\}
$$

and

$$
V_{N}=\left\{(x, y) \in X \times X: d\left(f^{n} x, f^{n} y\right) \leq \zeta \text { for all }|n| \leq N\right\} .
$$

Lemma 1. For every positive $\eta$ there exists an $N$ such that $V_{N} \subset B_{\eta}$.

Assume $\varepsilon \leq \zeta / 3$. Let $0<\eta \leq \zeta / 3$ and choose $N$ as in Lemma 1. Since the method $\varphi \in \mathcal{T}_{\text {c }}$ is continuous, there is a $\mu>0$ such that $d(x, y) \leq \mu$ implies $d\left(\varphi(x)_{n}, \varphi(y)_{n}\right) \leq \eta$ for all $|n| \leq N$. For $d(x, y) \leq \mu$ and $|n| \leq N$ we then have

$$
\begin{aligned}
d\left(f^{n} h(x), f^{n} h(y)\right) \leq & d\left(f^{n} h(x), \varphi(x)_{n}\right) \\
& +d\left(\varphi(x)_{n}, \varphi(y)_{n}\right)+d\left(\varphi(y)_{n}, f^{n} h(y)\right) \\
\leq & \varepsilon+\eta+\varepsilon \leq \zeta,
\end{aligned}
$$

so $(h(x), h(y)) \in V_{N} \subset B_{\eta}$. Hence, $h$ is continuous.

Setting $n=0$ in (1) we also have $d(h(x), x) \leq \varepsilon$ for all $x \in X$. From [8] it is known that a continuous map sufficiently close to the identity map on a compact manifold is surjective. Consequently, for $\varepsilon$ sufficiently small and $\delta$ chosen as above, the mapping $h=h_{\varphi}$ is onto for any $\delta$-method $\varphi \in \mathcal{T}_{\mathrm{c}}$. For any point $y \in X$ we can thus find an $x \in X$ such that $h(x)=y$. From inequality (1) we then have

$$
d\left(f^{n} y, \varphi(x)_{n}\right)=d\left(f^{n} h(x), \varphi(x)_{n}\right) \leq \varepsilon
$$

for all $n \in \mathbb{Z}$, which proves the inverse shadowing of $f$.

The proof of the second statement of the theorem is similar, so we leave the details to the reader; here we only note that we can choose $\beta_{1}>0$ to satisfy

$$
\alpha \beta_{1} \leq \min \left\{\alpha \beta, \frac{\zeta}{3}, \varepsilon_{0}\right\},
$$

where $\varepsilon_{0}$ is such that any continuous mapping $h: X \rightarrow X$ with $D_{\infty}\left(h, \mathrm{id}_{X}\right)$ $\leq \varepsilon_{0}$ is onto.

The assumption that the space is a manifold cannot be easily removed. We adapt the following counterexample from [14].

ExAmple 3 . Consider $X=\{0,1\}^{\mathbb{Z}}$ with a metric $d$ defined by $d(x, y)=$ $2^{-n}$ if $x_{0}=y_{0}$, where $n=\max \left\{k \geq 0: \forall|i|<k, x_{i}=y_{i}\right\}$, and by $d(x, y)=1$ 
otherwise. Let $f$ be the shift homeomorphism on $X$, i.e. with $(f x)_{n}=x_{n+1}$, and consider a sequence of methods $\varphi_{m}$ determined by mappings $g_{m}$ defined by

$$
g_{m}(x)_{i}= \begin{cases}x_{i} & \text { for }|i|>m, \\ x_{i+1} & \text { for } i=-m, \ldots, m-1, \\ x_{m} & \text { for } i=m,\end{cases}
$$

for $m=1,2, \ldots$ These mappings $g_{m}$ are homeomorphisms with $g_{m}^{2 m+1}=$ $\operatorname{id}_{X}$, while $\varphi_{m}$ is a $2^{-m}$-method with $\varphi_{m} \in \mathcal{T}_{\text {c }}$ for each $m=1,2, \ldots$

The shift mapping $f$ here is not inverse shadowing. To see this, let $\varepsilon=$ $1 / 2$ and pick $\delta$ arbitrarily and fix it. For $m$ with $2^{-m}<\delta$ the method $\varphi_{m}$ is a $\delta$-method. Define $x \in X$ by $x_{0}=1$ and $x_{i}=0$ for $i \neq 0$, and let $y \in X$. If $y_{0} \neq 1$, then inequality ( 1 ) is violated for $n=0$. On the other hand, if if $y_{0}=1$ then after $2 m+1$ iterations the sequence $f^{2 m+1} x$ has 0 as its 0 th component, while the sequence $g^{2 m+1} y=y$ has 1 as its 0 th component and so $d\left(f^{2 m+1} x, \varphi_{m}(y)_{2 m+1}\right)=1>\varepsilon$.

The shift mapping $f$ is, however, expansive (proof immediate) and shadowing [14]; in fact, it is $(2,1)$-shadowing.

4. Extensions. A homeomorphism $f$ on a compact metric space $X$ is topologically stable [12] if for every $\varepsilon>0$ there exists a $\delta>0$ such that for any homeomorphism $g$ on $X$ with $D_{\infty}(g, f) \leq \delta$ there exists a continuous surjective map $h$ on $X$ with $D_{\infty}\left(h, \mathrm{id}_{X}\right) \leq \varepsilon$ and $f \circ h=h \circ g$. Let $\mathcal{T}_{\mathrm{h}}$ be the class of methods generated by homeomorphisms, choose $\delta$ corresponding to $\varepsilon$ as in the above definition and a $\delta$-method $\varphi \in \mathcal{T}_{\mathrm{h}}$ and fix a point $y \in X$. If $g$ is a homeomorphism generating the method $\varphi$ and $x \in X$ is a point such that $h(x)=y$, then

$$
d\left(\varphi(x)_{n}, f^{n} y\right)=d\left(g^{n} x, f^{n} h(x)\right)=d\left(g^{n} x, h\left(g^{n} x\right)\right) \leq D_{\infty}\left(f, \operatorname{id}_{X}\right) \leq \varepsilon
$$

for all $n \in \mathbb{Z}$. Hence $f$ has the inverse shadowing property with respect to methods in the class $\mathcal{T}_{\mathrm{h}}$.

On the other hand, it is known $[12,14]$ that topological stability implies shadowing on a compact manifold, which we can combine with the preceding to conclude that topological stability on a compact manifold implies bishadowing with respect to the class $\mathcal{T}_{\mathrm{h}}$. Note, however, that $\mathcal{T}_{\mathrm{h}}$ is somewhat smaller than the class $\mathcal{T}_{\mathrm{c}}$ in Theorem 1 .

Theorem 1 is also true for expanding maps if we restrict ourselves to positive orbits and positive pseudo-orbits, i.e. sequences $\left\{x_{n}\right\}_{n \in \mathbb{N}} \in X^{\mathbb{N}}$, where $\mathbb{N}=\{0,1, \ldots\}$. A continuous surjection $f: X \rightarrow X$ of a compact metric space $X$ is called an expanding map if it is positively expansive and open $[1,13]$.

THEOREM 2. An expanding map on a compact manifold is bishadowing with respect to the class $\mathcal{T}_{\mathrm{c}}$. 
The proof follows from the fact that an expanding map is shadowing (see [11] for a proof based on the inverse limit space technique) and from the proof of Theorem 1 with some obvious changes to show that the map is also inverse shadowing, and hence bishadowing.

\section{References}

[1] N. Aoki, Topological Dynamics, in: Topics in General Topology, K. Morita and J. Nagata (eds.), Elsevier, 1989, 625-739.

[2] R. Corless and S. Pilyugin, Approximate and real trajectories for generic dynamical systems, J. Math. Anal. Appl. 189 (1995), 409-423.

[3] P. Diamond, P. E. Kloeden, V. Kozyakin and A. Pokrovskiǔ, Expansivity of semi-hyperbolic Lipschitz mappings, Bull. Austral. Math. Soc. 51 (1995), 301-308.

[4] - , - - - - Computer robustness of semi-hyperbolic mappings, Random Comput. Dynam. 3 (1995), 57-70.

[5] - - - - - , Robustness of observed behaviour of semi-hyperbolic dynamical systems, Avtomat. i Telemekh. 11 (1995), to appear (in Russian).

[6] P. Diamond, P. E. Kloeden and A. Pokrovskǐr, Shadowing and approximation in dynamical systems, in: CMA 3rd Miniconference on Analysis, G. Martin and H. B. Thompson (eds.), Proc. Centre Math. Appl. Austral. Nat. Univ. 33, Austral. Nat. Univ., Canberra, 1994, 47-60.

[7] R. Mañé, Ergodic Theory and Differentiable Dynamics, Springer, 1987.

[8] J. Munkres, Elementary Differential Topology, Princeton Univ. Press, 1963.

[9] J. Ombach, Equivalent conditions for hyperbolic coordinates, Topology Appl. 23 (1986), 87-90.

[10] -, Consequences of the pseudo-orbits tracing property and expansiveness, J. Austral. Math. Soc. Ser. A 43 (1987), 301-313.

[11] _, Expansive homeomorphisms with the pseudo orbits tracing property, preprint 383, Inst. Math. Polish Acad. Sci., 1987.

[12] S. Pilyugin, The space of Dynamical Systems with $C^{0}$-Topology, Lecture Notes in Math. 1571, Springer, 1991.

[13] D. Ruelle, Thermodynamic Formalism, Addison-Wesley, 1978.

[14] P. Walters, On the pseudo-orbit tracing property and its relationship to stability, in: Lecture Notes in Math. 668, Springer, 1978, 231-244.

School of Computing and Mathematics

Deakin University, Geelong Campus

Geelong, Victoria 3217

Australia

E-mail: kloeden@deakin.au.edu
Institute of Mathematics Polish Academy of Sciences Cracow Branch Św. Tomasza 30 31-027 Kraków, Poland E-mail: ombach@im.uj.edu.pl 